\title{
Massagem uterina pós-parto e eficiência reprodutiva em vacas nelore
}

\section{Effect of uterine manipulation on reproductive efficiency in nelore cows}

\author{
Christiana Fabricio de Noronha, Luis Altamiro Garcia Nogueira, Tânia Góes de Pinho, Flavio Vieites
}

\begin{abstract}
Resumo
Conduziu-se experimento em fazenda de pecuária de corte, objetivando verificar o efeito da massagem uterina sobre $o$ período parto primeiro cio observado, período de serviço e percentual de gestação em 152 vacas Nelore primíparas e pluríparas, com idade de 3 a 10 anos, com condição corporal, em média, de 6 pontos (escala de 1 a 9 ), paridas entre os meses de outubro de 1994 e fevereiro de 1995. As fêmeas foram separadas ao acaso, e submetidas a quatro tratamentos: T1 $(n=34)$, T2 $(n=39)$, T3 $(n=41)$, que receberam uma massagem uterina no $20^{\circ}, 30^{\circ}$ ou $40^{\circ}$ dia pós-parto (d.p.p.), respectivamente, e T4 $(n=38)$ controle, que não recebeu massagem uterina. O período de observação de cio foi de 130 dias e o percentual de gestação foi obtido após 300 d.p.p. (de outubro a julho). Os percentuais de fêmeas em estro, durante o período estudado, de acordo com os tratamentos 1, 2, 3 e 4, para primíparas e pluríparas, foram, respectivamente, zero e $52 \% ; 29,4 \%$ e $40,9 \% ; 17,6 \%$ e $29,1 \% ; 7,7 \%$ e $24,0 \%$ ( $P>0,05)$. As médias gerais do intervalo entre o parto e o $1^{2}$ estro, em dias, para primíparas e pluríparas foram, respectivamente, 97,33 $\pm 9,41$ e 96,23 $\pm 4,10$ $(P>0,05)$. O período de serviço e o percentual de gestação das vacas primíparas não foram analisados devido ao número reduzido de animais gestantes nesta ordem de parição. O sexo dos bezerros ao pé não afetou o período de serviço das vacas pluríparas $(P>0,05)$. As médias do período de serviço, em dias, e o percentual de gestação das vacas pluríparas, de acordo com os tratamentos 1, 2, 3 e 4 foram, respectivamente, 116,08 $\pm 10,94$ e $48 \%, 143,5 \pm 15,0$ e 54,5\%, 145,91 $\pm 17,7$ e $45,8 \%$ e $144,15 \pm 11,58$ e $52 \%$, ( $P>0,05)$.
\end{abstract}

Palavras-chave: zebu; massagem uterina; puerpério; eficiência reprodutiva.

\section{Introdução}

O intervalo entre partos constitui o parâmetro mais importante na avaliação da eficiência reprodutiva e tem a duração ideal de 365 dias. Para se reduzir o período de serviço é de fundamental importância um bom conhecimento dos mecanismos fisiológicos no período pós-parto, caracterizado pela involução uterina e restabelecimento da atividade ovariana (Nett, 1987; Pereira, 1996).

A relação entre involução uterina e eficiência reprodutiva está vinculada ao intervalo mínimo pós-parto para que os órgãos genitais estejam aptos a conceber (Vandemark \& Salisbury, 1950; Shannon et al., 1952). A pequena taxa de concepção antes dos 30 dias pós-parto está correlacionada com involução uterina incompleta (Moller, 1970).

A involução uterina é conseqüência da vasoconstricção e das contrações musculares e consiste em mudanças fisiomorfológicas dos tecidos, podendo ser dividida em: redução do tamanho do útero, perda de tecidos uterinos e reconstituição do tecido residual (Guilbault et al., 1984').

A involução uterina normal tem sido associada com a síntese maciça de PGF2a permanente do $7^{\circ}$ ao $15^{\circ}$ dia pós- parto (Guilbault et al., 1984a). Segundo Guilbault et al. $\left(1987^{b}\right)$, a involução uterina parece ser dependente tanto da duração da liberação de PGF2a, quanto da magnitude desta liberação. Guilbault et al. (1988) concluíram que a aplicação de PGF2a durante os primeiros 18 dias após o parto acelera a involução uterina, aumentando o tônus do útero. Estes pesquisadores sugerem, ainda, que a PGF2a seria um produto oriundo do processo de involução, e que, em parte, poderia agir no ovário, ajudando o restabelecimento da atividade ovariana.

Ainda não foi estabelecida uma base fisiológica para o uso de PGF2a no período imediato pós-parto, na ausência de uma estrutura lútea, nem os mecanismos pelos quais a liberação de PGF2a influencia o reinício da atividade ovariana (Benmrad \& Stevenson, 1986). Entretanto, as ovulações, diagnosticadas pelo aumento da concentração plasmática de progesterona, não ocorrem até que se cesse a liberação de PGF2a ou que a mesma atinja concentração basal. Sendo assim, a produção e/ou liberação de PGF2a poderia ter um papel muito complexo na restauração da atividade ovariana pós-parto. De acordo com Randel et al. (1996), quando a fêmea bovina está produzindo baixa concentração de estrógenos (anestro pós-parto), a 
hipófise tem uma habilidade aumentada de responder ao GnRH quando exposta primeiro à PGF2a.

Uma elevada concentração do metabólito da PGF2a (PGFM), é uma característica do período pós-parto na vaca, que alcança valores máximos entre $01^{10}$ e $04^{\circ}$ dia pósparto, e retorna progressivamente à concentração basal entre o $10^{\circ}$ e o 20(Guilbault et al., 1984b; Randel et al., 1986). Esta elevação pode ser explicada pela presença do tecido placentário materno por um período pós-parto limitado (Thatcher et al., 1982).

A supressão de PGF2a não altera a involução uterina mas reduz a atividade do ovário contralateral ao corno previamente gestante (Guilbault et al., 1987 a) concluindo, assim, que a PGF2a pode estar envolvida no recomeço da atividade ovariana pós-parto.

Tolleson \& Randel (1987) e Velez et al. (1991) afirmam que ocorre liberação endógena de PGF2a, no post-partum, devido à massagem uterina, e que esta liberação substitui os benefícios da aplicação exógena da mesma (Tolleson \& Randel, 1987). Randel et al. (1996) sugeriram que a ação da PGF2a no eixo hipófise-hipotalâmico realmente estimulou a liberação de LH.

Wann \& Randel (1990), Conciani (1991), Resende (1993) e Nogueira (1994) demonstraram que vacas primíparas não respondem a manipulação uterina. Foi verificado também, a ineficácia da manipulação uterina naquelas vacas pluríparas cuja involução uterina já era completa (Randel et al., 1986; Tolleson \& Randel, 1987 e Tolleson \& Randel, 1988). Ao contrário das primíparas, a massagem uterina em pluríparas parece ter um efeito estimulatório. Randel et al. (1996) confirmam que a manipulação uterina ou a PGF2a exógena reduz o período pós-parto em vacas pluríparas. A ordem do parto influenciou significativamente o intervalo de partos, sendo maior no primeiro intervalo, decrescendo subseqüentemente nos demais (Balieiro et al., 1981).

Um dos fatores mais influentes na atividade ovariana durante o período inicial pós-parto é a amamentação, resultando num intervalo entre partos maior (Eduvie \& Dawuda, 1986). Porém, não só a amamentação, como também a presença do bezerro e a própria glândula mamária exercem um efeito inibitório sobre a função reprodutiva (Viker et al., 1989). Wagner \& Oxenreider (1972) verificaram que as concentrações de corticóides no plasma de vacas amamentando foi maior que nas vacas sem bezerro, lactando ou não, e sugerem que o aumento na atividade da adrenal pode exercer um efeito depressivo na produção hipotalâmica de $\mathrm{GnRH}$.

\section{Material e métodos}

O experimento foi realizado nas Fazendas Consorciadas FC, município de Guapimirim, no Estado do Rio de Janei- ro, em duas fases experimentais, durante o período compreendido entre outubro de 1994 e julho de 1996.

Os animais foram mantidos em regime de manejo a campo, com pastagem predominante de Brachiaria mutica (capim-angola), com água e sal mineral ad libitum.

Foram utilizadas fêmeas da raça Nelore primíparas $(n=56)$ e pluríparas $(n=96)$, recém-paridas, com bezerro ao pé, e idade entre três e dez anos e que não apresentaram parto distócico e/ou retenção de placenta e que até a ocasião do experimento não haviam retornado a atividade cíclica.

A condição corporal dos animais no início do experimento foi em média de 6, utilizando-se da escala de 1 (muito magra) a 9 (muito gorda) (Richards et al., 1986).

Os 152 animais pariram de outubro a fevereiro de cada ano e foram distribuídos ao acaso nos seus devidos grupos (primíparas e pluríparas) . Os grupos foram submetidos a quatro tratamentos:

T1 - 9 vacas primíparas e 25 vacas pluríparas, que receberam uma leve massagem uterina por 40 segundos aos 20 dias pós-parto;

T2 - 17 primíparas e 22 vacas pluríparas, que receberam a massagem como no T1, aos 30 dias pós-parto;

T3 - 17 primíparas e 24 vacas pluríparas, que receberam a massagem como no T1, aos 40 dias pós-parto; e

T4 - 13 primíparas e 25 vacas pluríparas, correspondendo ao grupo testemunha não receberam massagem uterina.

A detecção do estro foi realizada por um período de 130 dias pós-parto. Uma vez em cio, as vacas foram inseminadas de 10 a 12 horas após a detecção do estro e, posteriormente, o diagnóstico de gestação foi realizado por palpação retal entre os dias 30 e 60 após as inseminações.

Para a análise estatística foram calculadas médias aritméticas e seus respectivos erros-padrão para os períodos do parto-1을 estro das vacas primíparas e pluríparas e períodos de serviço das vacas pluríparas.

Para comparação dos tratamentos 1, 2, 3 e 4 e ordens de parição (para o intervalo parto $-1^{\circ}$ estro) foi empregado o teste "F" de Brieger, em análise de variância.

$\mathrm{Na}$ associação dos percentuais de freqüências de estro e ordens de parição, foi utilizado o teste não-paramétrico de qui-quadrado.

Para comparação do percentual de estros das ordens de parição por tratamento e do percentual de gestação entre os tratamentos das vacas pluríparas, foi aplicado o teste " $Z$ ".

O modelo estatístico utilizado no estudo do intervalo parto-1e estro observado, de vacas primíparas e pluríparas foi: 
Yijk $=\mu+\mathrm{Gi}+\mathrm{OPj}+\mathrm{eijk}$ onde,

Yijk $=$ resposta da késima vaca do tratamento $i$, da ordem do parto j para a característica estudada

$\mu=$ média geral

$\mathbf{G i}=$ efeito do tratamento $i$, sendo $i=1,2,3$ e 4

$\mathrm{OPj}=$ efeito da ordem de parição $\mathrm{j}$ (primípara ou multípara), sendo $\mathrm{j}=1$ e 2

Eijk = erro aleatório associado a cada observação j e $k$ de cada combinação dos fatores acima.

O modelo estatístico utilizado no estudo do período de serviço e influência do sexo dos bezerros ao pé em vacas pluríparas foi:

Yijk $=\mu+G i+S j+$ eijk onde,

Yijk = resposta da késima vaca do tratamento $i$, e do sexo do bezerro ao pé j para a característica estudada

$\mu=$ média geral

$\mathbf{G i}=$ efeito do tratamento $i$, sendo $i=1,2,3$ e 4

$\mathrm{S} \mathbf{j}=$ efeito do sexo do bezerro ao pé, sendo $\mathbf{j}=1$ e 2

eijk = erro aleatório associado a cada observação de cada combinação dos fatores acima.

\section{Resultados e discussão}

Dados relativos às médias de intervalo em dias para o intervalo entre parto e primeiro estro são apresentados na Tabela 1.

Tabela 1: Médias dos quadrados mínimos e respectivos erros-padrão (MQM $\pm P P$ ), observado em vacas primíparas e pluríparas, para o intervalo em dias, entre o parto e o primeiro estro

\begin{tabular}{lc}
\hline Ordem de parição $(n)$ & $M Q M \pm E P$ \\
\hline Primíparas (09) & $97,33 \pm 9,41$ \\
Pluríparas (35) & $96,23 \pm 4,1$ \\
\hline
\end{tabular}

As médias gerais obtidas em dias do período parto- $1^{\circ}$ cio entre as vacas primíparas e pluríparas (Tabela 1) quando comparadas, não diferem entre si $(P>0,05)$. As vacas pluríparas apresentaram um período de anestro relativamente longo quando comparado aos resultados obtidos por Nogueira (1994), em vacas da mesma ordem de parição, que foi de $43,3 \pm 12,0$ dias, porém bem semelhante aos $94,5 \pm 33,5$ dias observados pelo mesmo pesquisador nas vacas primíparas. Algumas vacas, embora primíparas, tinham a idade e a condição corporal próximas das pluríparas, o que pode ter contribuído nas aproximações dos resultados obtidos.

Houve um pequeno declínio na condição corporal média das vacas em menos de 1 ponto na escala de 1 a 9 (Richards, 1986) durante o período pós-parto. Essa queda, significando um balanço energético negativo, pode ter sido um fator de interferência, atrasando a atividade ovariana (Butler \& Smith, 1989).

Inúmeros outros fatores também podem ter influenciado a duração do período parto-1²io, retardando o reinício da atividade ovariana ou influenciando o período de anestro, aproximando as médias dos resultados verificados entre os tratamentos. Dentre eles estão o estresse calórico, condição corporal, amamentação e presença do bezerro conforme citado por Wagner \& Oxenreider (1972).

Os percentuais de cio observados, de acordo com os tratamentos, de vacas primíparas e pluríparas, encontram-se na Tabela 2.

Tabela 2: Número de vacas primíparas e pluríparas e respectivos percentuais de cios observados até $130 \mathrm{dpp}$, de acordo com os tratamentos

\begin{tabular}{ccc}
\hline Tratamento & Primípara n(\%) & Plurípara n(\%) \\
\hline 1 & $9(0,0)$ & $25(52,0)$ \\
2 & $17(29,4)$ & $22(40,9)$ \\
3 & $17(17,6)$ & $24(29,1)$ \\
4 & $13(7,7)$ & $25(24,0)$ \\
\hline
\end{tabular}

As análises estatísticas mostram não haver diferenças entre os tratamentos dentro das categorias de vacas, para o percentual de cio observados nos 130 dias pós-parto $(P>0,05)$.

Em relação às vacas primíparas, estes resultados são semelhantes aos obtidos por vários autores como Tolleson \& Randel (1987), Wann \& Randel (1990), Conciani (1991), Resende (1993) e Nogueira (1994), que também não obtiveram, no período puerperal, resposta às massagens uterinas em vacas desta categoria. Entretanto, contrariando os resultados do presente trabalho, Guilbault et al. (1984a), Tolleson \& Randel (1987), verificaram que a massagem uterina no pós-parto, em vacas pluríparas, estimulou a síntese e liberação de PGF2a, substituindo os benefícios da aplicação exógena da mesma.

Observa-se uma tendência de maior percentual de cios nas vacas pluríparas massageadas, em relação ao grupo testemunha. Esta diferença não foi significativa $(P>0,05)$, contrariando as citações de Randel et al. (1986), Tolleson \& Randel (1987) e Tolleson \& Randel (1988) que verificaram a eficácia da manipulação uterina, nas vacas pluríparas cuja involução uterina não era completa.

As médias dos quadrados mínimos e respectivos errospadrão, em dias, para o período de serviço, e respectiva taxa de gestação, durante a fase experimental de 300 dias, das vacas pluríparas, é mostrado na Tabela 3 . O número de vacas primíparas gestantes foi pequeno e não considerado nas análises. 
Tabela 3: Média dos quadrados mínimos e respectivos erros-padrão (MQM \pm EP) , em dias, para o período de serviço, e percentual de gestação, por tratamento, em vacas pluríparas, durante a fase experimental de 300 dias

\begin{tabular}{cccc}
\hline Tratamento & $\mathrm{n}$ & \multicolumn{1}{c}{$\mathrm{MQM} \pm \mathrm{EP}$} & \%Gestação \\
\hline 1 & 12 & $116,08 \pm 10,94$ & 48,0 \\
2 & 12 & $143,50 \pm 15,0$ & 54,5 \\
3 & 11 & $145,91 \pm 17,71$ & 45,8 \\
4 & 13 & $144,15 \pm 11,58$ & 52,0 \\
\hline
\end{tabular}

É citado que a manipulação uterina promove liberação de PGF2a e que somente nas vacas pluríparas ocorre uma redução do período de serviço (Benmrad \& Stevenson, 1986; Tolleson \& Randel, 1987; Randel et al., 1990; Velez et al., 1991 e Randel et al., 1996). Entretanto, no presente experimento não foram observadas diferenças $(p>0,05)$, entre os tratamentos, nas vacas pluríparas, em relação ao período de serviço e percentual de gestação. Neste aspecto, todas as vacas pluríparas deste experimento constituíram problema, apresentando mais de 100 dias de período de serviço.
A taxa de gestação não apresentou diferença $(P>0,05)$ entre os diferentes tratamentos. Diversos autores (Guilbault et al.,1987a, Guilbault et al.,1987b e Guilbault et al.,1988), indicam haver uma relação entre à involução uterina e o restabelecimento da atividade ovariana. Embora observações relativas a involução uterina não tenham sido objetivo desse trabalho, esta pode ter sido responsável pelo elevado percentual de anestro e pelo atraso no retorno a atividade cíclica ovariana, contribuindo certamente com os resultados observados.

Outro fator como o sexo dos bezerros ao pé, avaliado nas vacas pluríparas, não foi significativo $(p>0,05)$. Resultados semelhantes foram obtidos por Andrade (1977), embora Balieiro et al. (1981) tenham verificado influência deste fator.

\section{Conclusões}

As massagens uterinas não foram efetivas na diminuição do período entre o parto e o $1^{2} \mathrm{cio}$, em vacas Nelore, tanto primíparas quanto pluríparas.

As vacas pluríparas submetidas às massagens uterinas aos 20, 30 ou 40 dias pós-parto, não tiveram o período de serviço reduzido, nem a taxa de prenhez alterada.

\begin{abstract}
From 1994 to 1995 , the effect of uterine manipulation on interval from parturition to the first estrus, parturition to conception and pregnancy rate was studied.We used 152 Nelore primiparous heifers and cows aging from 3 to 10 years old. The average body score was six (total range 1 to 9). All parturition occured between October and February. The females were randomly divided into four different treatments. Cows from T1 $(n=34)$, T2 $(n=39)$, T3 $(n=41)$ were slightely manipulated per retum for 40 seconds on day $20, d 30$ and d 40 postpartum, respectively. Cows from T4 ( $n=38$ ) were control group (without massage). First postpartum estrus was observed until day 130 postpartum (pp), and pregnancy rate was obtained until day $300 \mathrm{pp}$ (from October to July). The percentual of females showing estrus during period studied was zero and $52,0 \%$; $29,4 \%$ and $40,9 \% ; 17,7 \%$ and $29,1 \% ; 7,7 \%$ and $24,0 \%$, for primiparous and pluriparous, in treatments $1,2,3$ and 4 , respectively. The average interval between the parturition and the first estrus of primiparous and pluriparous, in days, were, respectively, $97,33 \pm 9,41$ and $96,23 \pm 4,10$. The interval from parturition to conception and pregnancy rate of primiparous was not analysed because the rate of pregnancies was reduced in this animals. The averages interval from parturition to conception and pregnancy rate of pluriparous, in days, according to treatments $1,2,3$ and 4 were, respectively, $116,08 \pm 10,94$ and $48 \%, 143,5 \pm 15,0$ and $54,5 \%, 145,91 \pm 17,7$ and $45,8 \%$ and $144,15 \pm 11,58$ and $52 \%$ and did not differ between themselves. The treatments did not affect the reproductive efficiency, in Nelore cows. $(P>0,05)$.
\end{abstract}

Keywords: zebu; uterine manipulation; postpartum; reproductive efficiency.

\section{Referências bibliográficas}

ANDRADE V.J., TORRES J.R., CARNEIRO G.G. et al. Idade à primeira parição e intervalo entre partos num rebanho Guzerá na área do serrado em Minas Gerais. Arquivos da Escola de Veterinária da UFMG, v. 29, n.1, p.85-88, 1977.

BALIEIRO E.C., SILVA H.M., CARNEIRO G.G. et al. Eficiência reprodutiva de um rebanho Guzerá para leite. Arquivos da Escola de Veterinária da UFMG, v. 33, n. 3, p. 489-495, 1981.
BENMRAD M., STEVENSON J.S. Gonadotropin-releasing hormone and prostaglandin F2a for postpartum dairy cows: Estrous, ovulation and fertility traits. J. Dairy Sci., Champaign, v. 69 , n. 3, p. $800-811,1986$.

BUTLER, W.R., SMITH, R.D. Interrelationships between energy balance and postpartum reproductive function in dairy cattle. J. Dairy Sci., v. 72, n. 3, p. 767-783, 1989.

CONCIANI A.C. Efeito da massagem uterina associada ou não ao desmame temporário, sobre o desempenho reprodutivo de vacas de corte primíparas. Belo Horizonte, 1991. 57p. 
Tese (Mestrado em Zootecnia): Escola de Veterinária da Universidade Federal de Minas Gerais.

EDUVIE, L.O., DAWUDA, P.M. Effect of suckling on reproductive activities of Bunaji cows during the postpartum period. $J$. Agric. Sci., v. 107, n. 2, p. 235-238, 1986.

GUILBAULT L.A., VILLENEUVE P., DUFOUR J.J. Failure of exogenous prostaglandin $\mathrm{F} 2 \mathrm{a}$ to enhance uterine involution in beef cows. Canadian J. Anim. Sci., v. 68, n. 3, p. 669-676, 1988.

GUILBAULT L.A., THATCHER W.W., DROST M., HOPKINS S.M. Source of $F$ series prostaglandins during the early postpartum period in cattle. Biol. Reprod., v. 31, n. 5, p. 879$887,1984^{\mathrm{a}}$.

GUILBAULT L.A., THATCHER W.W., FOSTER, D.B. et al. Relationship of 15-keta-13, 14-dihydroprostaglandin F2a concentrations in peripheral plasma with local uterine production of $F$ series prostaglandins and changes in uterine blood flow during the early postpartum period of cattle. Biol. Reprod., v. 31, n. 5, p. 870-878, $1984^{\text {b. }}$.

GUILBAULT L.A., THATCHER W.W., DROST M. et al. Influence of a physiological infusion of prostaglandinF2a into postpartum cows with partially supressed endogenous production of prostaglandins. 1. Uterine and ovarian morfological responses. Theriogenology, v. 27, n. 6, p. 931946, 1987a'.

GUILBAULT L.A, THATCHER W.W., WILCOX, C.J. Influence of a physiological infusion of prostaglandin $\mathrm{F} 2 \mathrm{a}$ into postpartum cows with partially supressed endogenous production of prostaglandins. 2. Interrelationship of hormonal, ovarian and uterine responses. Theriogenology, v. 27, n. 6 , p. $947-957,1987^{\circ}$.

MOLLER, K. Uterine involution and ovarian activity after calving. New Zealand Vet. J., v. 18, p. 140-145, 1970.

NETT, T.M. Function of the hypotalamic-hypophisial axis during the postpartum period in ewes and cows. J. Reprod. Fertil. Suppl., n. 34, p. 201-213, 1987.

NOGUEIRA L.A.G. Alguns parâmetros clínicos e endócrinos relacionados ao retorno à atividade cíclica ovariana em vacas Bos taurus indicus. Belo Horizonte, 1994. 127p. Tese (Doutorado em Ciência Animal): Escola de Veterinária da Universidade Fedral de Minas Gerais, 1994.

PEREIRA, J.C.C. Melhoramento Genético Aplicado à Produção Animal. Belo Horizonte: [s.n.], 1996. 416 p. cap. 14: Melhoramento genético das raças zebus, p.189-198.

RANDEL, R.D., TOLLESON, D.R., PROCTOR, G.L. et al. Effects of alfaprostol and uterine palpation during postpartum period of Brangus cows. J. Anim. Sci. Suppl., v. 63, n. 1, p. 371, 1986.

RANDEL R.D. Nutrition and postpartum rebreeding in cattle. J. Anim. Sci. Suppl.,v. 68, n. 1, p. 13, 1990.
RANDEL R.D., LAMMOGLIA A W., LEWIS D.A. et al. Exogenous PGF2a enhanced $\mathrm{GnRH}$-induced $\mathrm{LH}$ release in postpartum cows. Theriogenology, v. 45, n. 3, p. 643-654, 1996.

RESENDE H.R.A. Efeito da amamentação e da massagem uterina sobre o desempenho reprodutivo de vacas primíparas Zebu. Belo Horizonte, 1993. 101p. Tese (Mestrado em Zootecnia), Escola de Veterinária da Universidade Federal de Minas Gerais.

RICHARDS M.W., SPITZER J.C., WARNER M.B. Effect of varying levels of postpartum nutrition and body conditionat calving on subsequent reproductive performance in beef cattle. $J$. Anim. Sci., v. 62, n. 2, p. 300-306. 1986.

SHANNON, F.P., SALISBURY, G.W., VANDEMARK, N.L. The fertility of cows inseminated at various intervals after calving. J. Animal Sci., v. 11, p. 355, 1952.

THATCHER, W.W., GUILBAULT, L.A., COLLIER, R.J. et al. The impact of antipartum physiology on postpartum performance in cows. In: KARG, H. \& SCHALLENBERGER, E. Factors influencing fertility in the postpartum cow. Hague: Martinus Nijhoff Publishers, 1982. p. 3-25.

TOLLESON D.R., RANDEL R.D. Physical manipulation of the postpartum bovine uterus and the subsequent release of prostaglandin F2a. J. Anim. Sci. Suppl., v. 65, n. 1, abstr. 523, 1987.

TOLLESON D.R., RANDEL R.D. Effects of alfaprostol and uterine palpation on postpartum interval and pregnancy rate to embryo transfer in Brahman influenced beef cows. Theriogenology, v. 29, n. 3, p. 555-564, 1988.

VANDEMARK,N.L., SALISBURY, G.W. The relation of the postpartum breeding interval to reproductive efficiency in the dairy cow. J. Animal Sci., v. 9, p. 307, 1950.

VELEZ J.S., RANDEL R.D., NEVENDORFF D.A. Effect of uterine manipulation on postpartum fertility and plasma 13, 14dihydro-15-keto-prostaglandin $\mathrm{F} 2 \mathrm{a}$ in Brahman cows and first calf heifers. Theriogenology, v. 36, n. 6, p. 987-998, 1991.

VIKER, S.D., McGUIRE, W.J., WRIGHT, J.M. et al. Cow-calf association delays postpartum ovulation in mastectomized cows. Theriogenology, v. 32, n. 3, p. 467-474, 1989.

WAGNER W.C., OXENREIDER S.L. Adrenal function in the cow. Diurnal changes and the effects of lactation and neurohypophysial hormones. J. Anim. Sci., v. 34, n. 4, p. 630-635, 1972.

WANN R.A.,RANDEL R.D. Effectof uterine manipulation 35 days after parturition on plasma concentrations of 13, 14dihydro-15-keto-prostaglandin $\mathrm{F} 2 \mathrm{a}$ in multiparous and primiparous Brahman cows. J. Anim. Sci., v. 68, n. 5, p. 1389-1394,1990. 